\title{
Beyond one-electron reaction in Li cathode materials: designing $\mathrm{Li}_{2} \mathrm{Mn}_{\mathrm{x}} \mathrm{Fe}_{1-\mathrm{x}} \mathrm{SiO}_{4}$
}

\author{
Anton Kokalj, Robert Dominko, Gregor Mali, Anton Meden, Miran Gaberscek, ${ }^{*}$ Janez Jamnik
}

Text S1. In $\mathrm{Li}_{2} \mathrm{MnSiO}_{4}$ the oxidation of $\mathrm{Mn}^{\mathrm{II}}$ ion, which has a stable half-filled $\mathrm{d}^{5}$ shell, requires a high energy penalty. By contrast, in $\mathrm{Li}_{2} \mathrm{FeSiO}_{4}$ the oxidation of $\mathrm{Fe}^{\mathrm{II}}$ with a less stable $\mathrm{d}^{6}$ configuration, into a stable $\mathrm{d}^{5}$ configuration of $\mathrm{Fe}^{\mathrm{III}}$ that appears formally in $\mathrm{LiFeSiO}_{4}$, is more facile. For these reasons, the SEC for the Mncompounds with more than $50 \%$ of lithium is larger than for the corresponding Fe-compounds. However, further oxidation of stable $\mathrm{Fe}^{\mathrm{III}}$ is more difficult. In the SEC curve (Fig 5a, see main article) this is seen as an abrupt, over 1.5 eV-jump, as we move from $\mathrm{LiFeSiO}_{4}$ to $\mathrm{Li}_{0.5} \mathrm{FeSiO}_{4}$. Here it is worth mentioning that $\mathrm{Li}_{2} \mathrm{MnSiO}_{4}$ and the $50 \%$ delithiated $\mathrm{LiFeSiO}_{4}$ are isoelectronic. The GGA-predicted band gaps are 2.4 and $1.6 \mathrm{eV}$, respectively, while the GGA+ $\mathrm{U}^{1}$ gives the band gaps of $\sim 4.4$ and $\sim 2.4 \mathrm{eV}$, respectively. Large band gaps are due to a half filled $\mathrm{d}^{5}$ shell configurations of constituent $\mathrm{Mn}^{\mathrm{II}}$ and $\mathrm{Fe}^{\mathrm{III}}$ ions. ${ }^{1}$ For all other $\mathrm{Li}$ concentrations the GGA predicted band gaps are smaller or vanishing (Fig. S5). Note, however, that in many cases GGA either severely underestimates band gaps or even produces a qualitatively wrong metallic ground state for materials containing (3d) transition metal ions. ${ }^{2-4}$ It has been argued that a low intrinsic electronic conductivity of $\mathrm{LiFeSiO}_{4}$ may also pose a limitation — in addition to the large voltages as discussed in this work — to extraction of more than one Fe ion/FU. ${ }^{5}$

1. Arroyo-de Dompablo, M. E.; Armand, M.; Tarascon, J.-M.; Amador, U. Electrochem. Commun. 2006, 8, 1292.

2. Anisimov, V. I.; Zaanen, J.; Andersen, O. K. Phys. Rev. B 1991, 44, 943.

3. Cococcioni, M.; de Gironcoli, S. Phys. Rev. B 2005, 71, 035105.

4. Zhou, F.; Kang, K.; Maxisch, T.; Ceder, G.; Morgan, D. Solid State Commun. 2004, 132, 181.

5. Larsson, P.; Ahuja, R.; Nyten, A.; Thomas, J. O. Electrochem. Commun. 2006, 8, 797. 
Text S2. In order to estimate the entropy contribution to formation free energies at finite temperatures, we use the following thermodynamic relation:

$$
T\left(S_{T}-S_{0}\right)=T \int_{0}^{T} C_{P}(T) / T d T
$$

where $C_{P}$ is the heat capacity, $S_{T}$ is the entropy at temperature $T$, whereas $S_{0}$ is the residual entropy at $0 \mathrm{~K}$. Because our experimental setup allows to measure $C_{P}$ only at room temperature or higher, we estimate the $C_{P}$ value at lower temperatures in a Debye-like manner $\left(C_{P} \propto T^{3}\right)$. More specifically, we fitted the experimental results to a polynomial, where we discarded the linear term, because it would correspond to a contribution of conducting electrons to $C_{P}$, while our compounds are not metallic (see Fig. 1a in the main article). Therefore we used: $C_{P}(T)=a T^{2}+b T^{3}$ (note that our treatment neglects any (magnetic) phase-transition below the room temperature). In Fig. S6, we show the $C_{P}$ data for $\mathrm{Li}_{2} \mathrm{MnSiO}_{4}$ together with the corresponding fit and the resulting $T S$ contribution, i.e., the curve corresponding to Eq. (S1). As evident from the figure, the $T\left(S_{T}-S_{0}\right)$ contribution at room temperature is about $1 / 4 \mathrm{eV} / \mathrm{FU}$. For the $\mathrm{Li}_{2} \mathrm{FeSiO}_{4}$ we have obtained a similar result (not shown).

As for the residual entropy at $0 \mathrm{~K}, S_{0}$, we assume it should be zero (or fairly small) for the end-members (i.e., $0 \%$ and $100 \%$ of $\mathrm{Li}$ ), whereas for the intermediate $\mathrm{Li}$ concentrations there might be configurational freedom even at $0 \mathrm{~K}$. Actually, the ideal configurational entropy would be $S^{\text {id }}{ }_{\text {conf }}=-k_{\mathrm{B}}[y \ln y+(1-y) \ln (1-y)]$, where $y(1-$ $y$ ) is the $\mathrm{Li}$ (vacancy) concentration. For the $50 \% \mathrm{Li}$ concentration, $S^{\text {id }}$ conf would be $2 \times 6 \cdot 10^{-5} \mathrm{eV} / \mathrm{K} / \mathrm{FU}$ (we used the factor of 2 because there are $2 \mathrm{Li}$ ions/FU). At room temperature, the corresponding $T S^{\text {id }}$ conf would be $\sim 0.04$ $\mathrm{eV} / \mathrm{FU}$. As our estimation of the $T\left(S_{T}-S_{0}\right)$ term is quite rough, we may neglect the residual entropy contribution, therefore the $T S_{T}$ is on the order of $1 / 4 \mathrm{eV} / \mathrm{FU}$. 


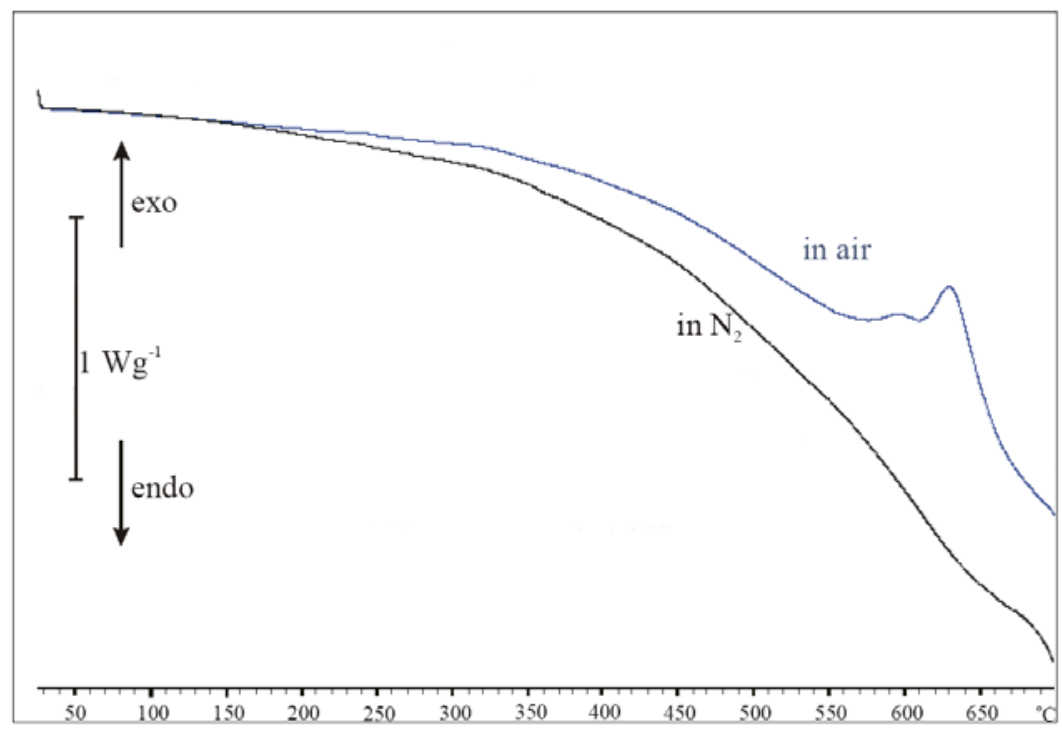

Figure S1. Differential scanning calorimetry of $\mathrm{Li}_{2} \mathrm{MnSiO}_{4}$. The material can be regarded thermally stable up to more than $500^{\circ} \mathrm{C}$. 


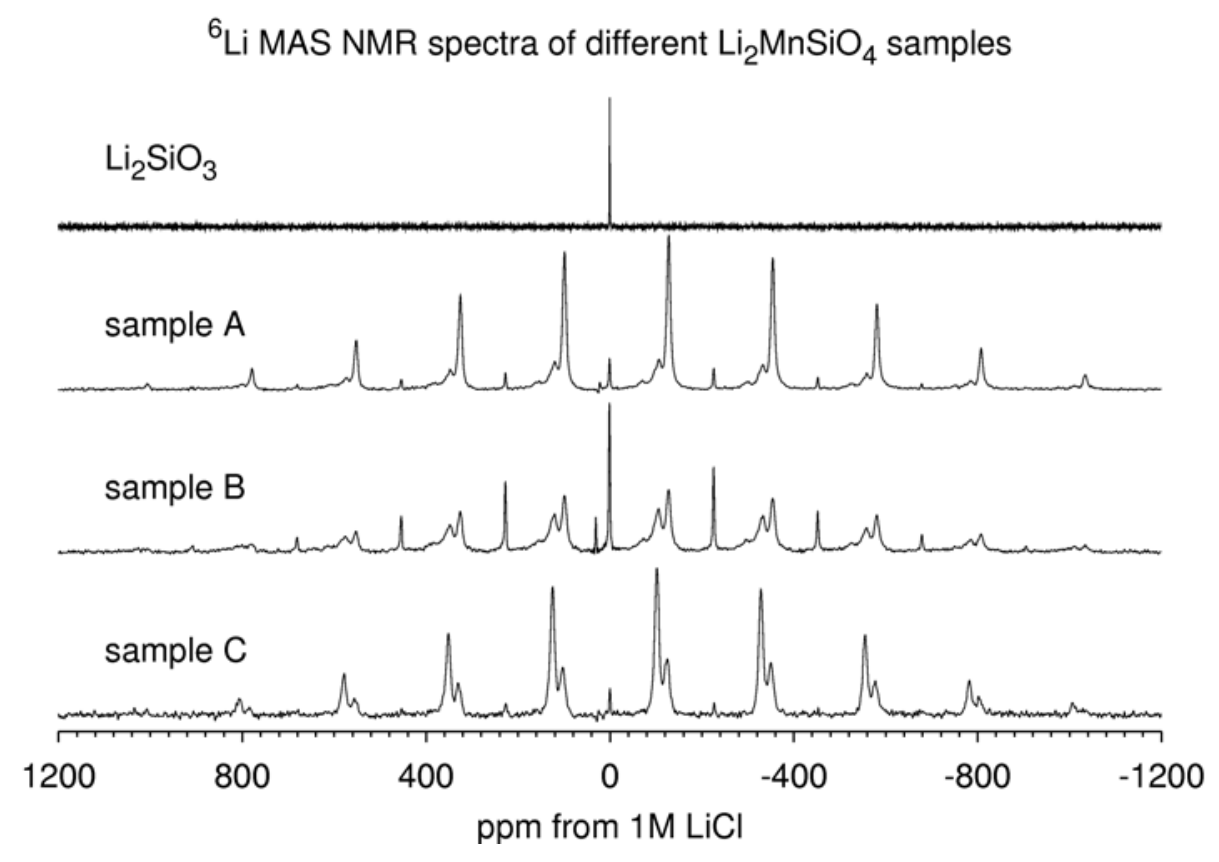

Figure S2. ${ }^{6} \mathrm{Li}$ MAS NMR spectra of $\mathrm{Li}_{2} \mathrm{SiO}_{3}$ and of different $\mathrm{Li}_{2} \mathrm{MnSiO}_{4}$ samples. Sample A was used in Rietveld analysis (see [ref 2] in the main article), which revealed a structural disorder in the $\mathrm{Li}_{2} \mathrm{MnSiO}_{4}$ material. Samples B and C were prepared using the same synthesis procedure, except that the former was cooled to room temperature in about 10 min while the cooling of the latter took about $1.5 \mathrm{~h}$. Sample $\mathrm{C}$ was used in this work for studying the effect of charge-discharge cycling, while sample B was used for studying the effect of Li extraction only (its NMR spectra are presented in Figure S3 below). 

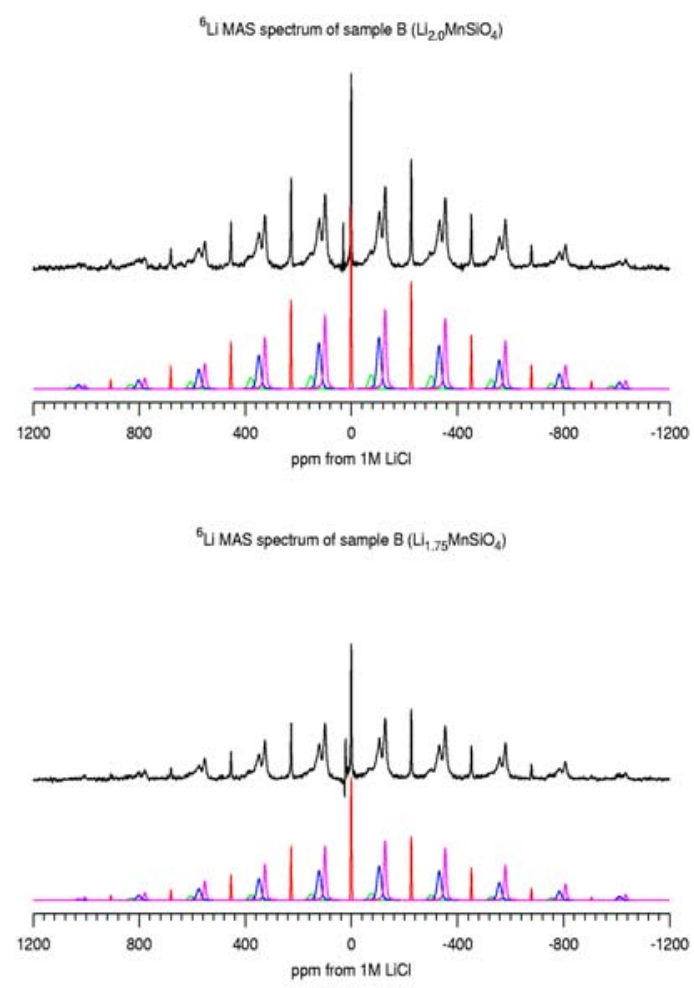

[Li MAS spectrum of sample $\mathrm{B}\left(\mathrm{U}_{1,0} \mathrm{MnSiO}_{4}\right)$

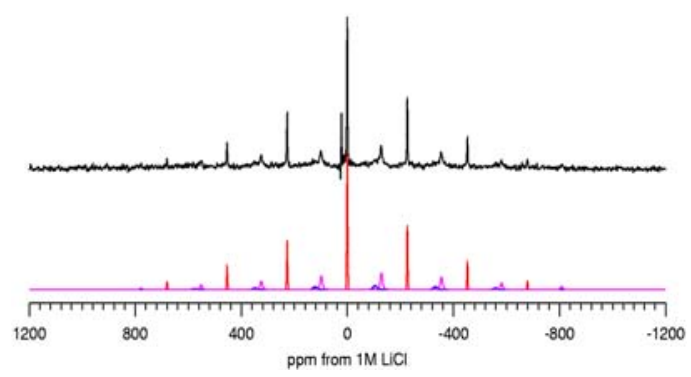

Figure S3. ${ }^{6} \mathrm{Li}$ MAS NMR spectra of $\mathrm{Li}_{2} \mathrm{MnSiO}_{4}$ (sample B) at different stages of Li extraction. The spectra show that only the $\mathrm{Li}$ ions that contribute to NMR peaks with isotropic shift between -70 and $-130 \mathrm{ppm}$ are extracted, and that the Li ions that contribute to the NMR peak with isotropic shift of about $0 \mathrm{ppm}$ remain within the material (they could either occupy a non- $\mathrm{Li}$ site in $\mathrm{Li}_{2} \mathrm{MnSiO}_{4}$ or belong to a paramagnetic Li-Mn impurity). Note also that a very broad 'background' signal starts to appear (and to increase) when progressively more Li is extracted (this is only seen when a detailed decomposition of the spectra is performed). 


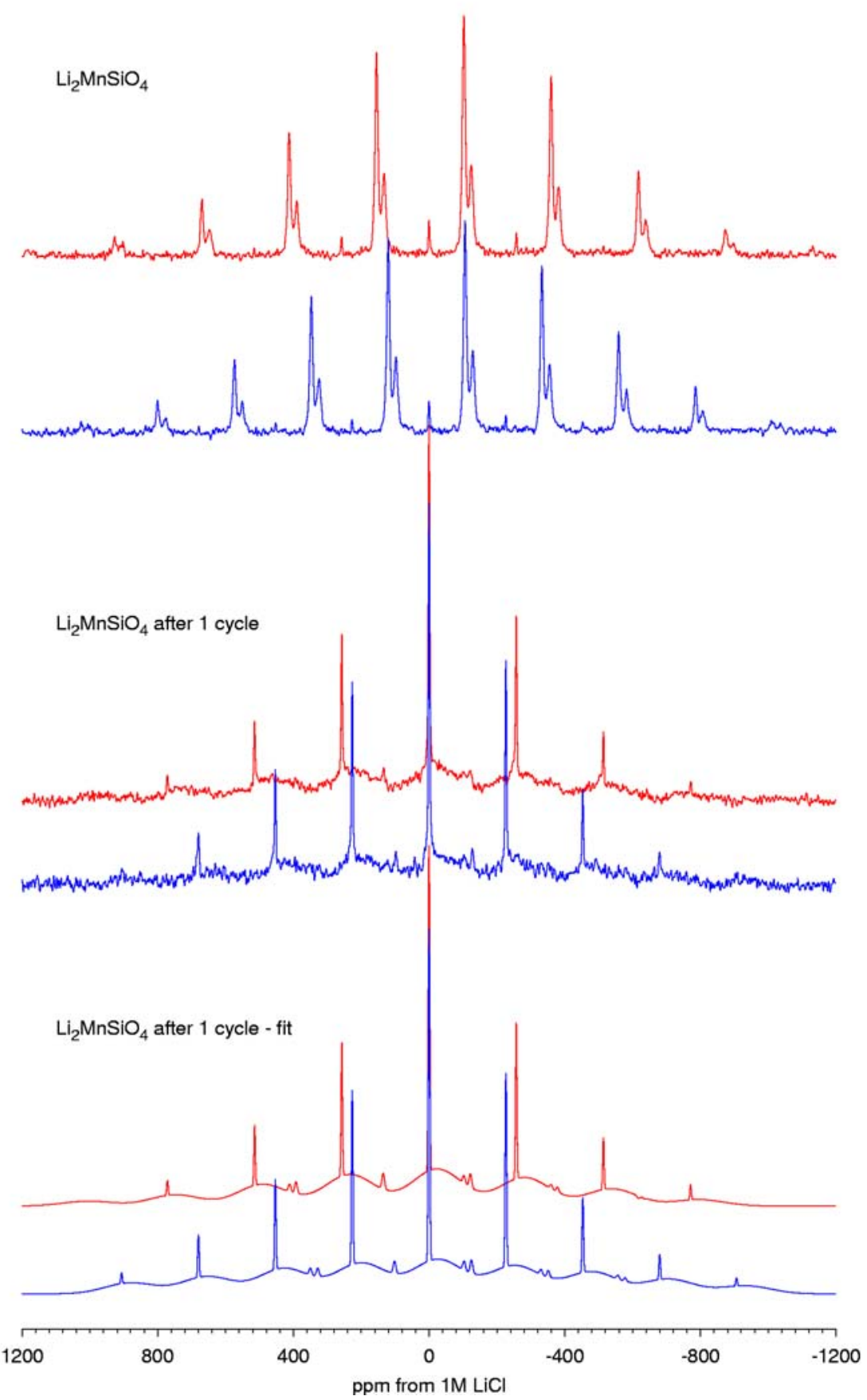

Figure S4. ${ }^{6} \mathrm{Li}$ MAS NMR spectra of $\mathrm{Li}_{2} \mathrm{MnSiO}_{4}$ sample C before and after a completed charge-discharge cycle recorded at two different sample rotation frequencies $(20000 \mathrm{~Hz}$ and $22730 \mathrm{~Hz}$ ). These spectra enable identification of isotropic bands and spinning sidebands. For a reliable identification of a centerband of a broad component in the spectrum of the cycled material, a decomposition (a fit) of the spectra was necessary. 

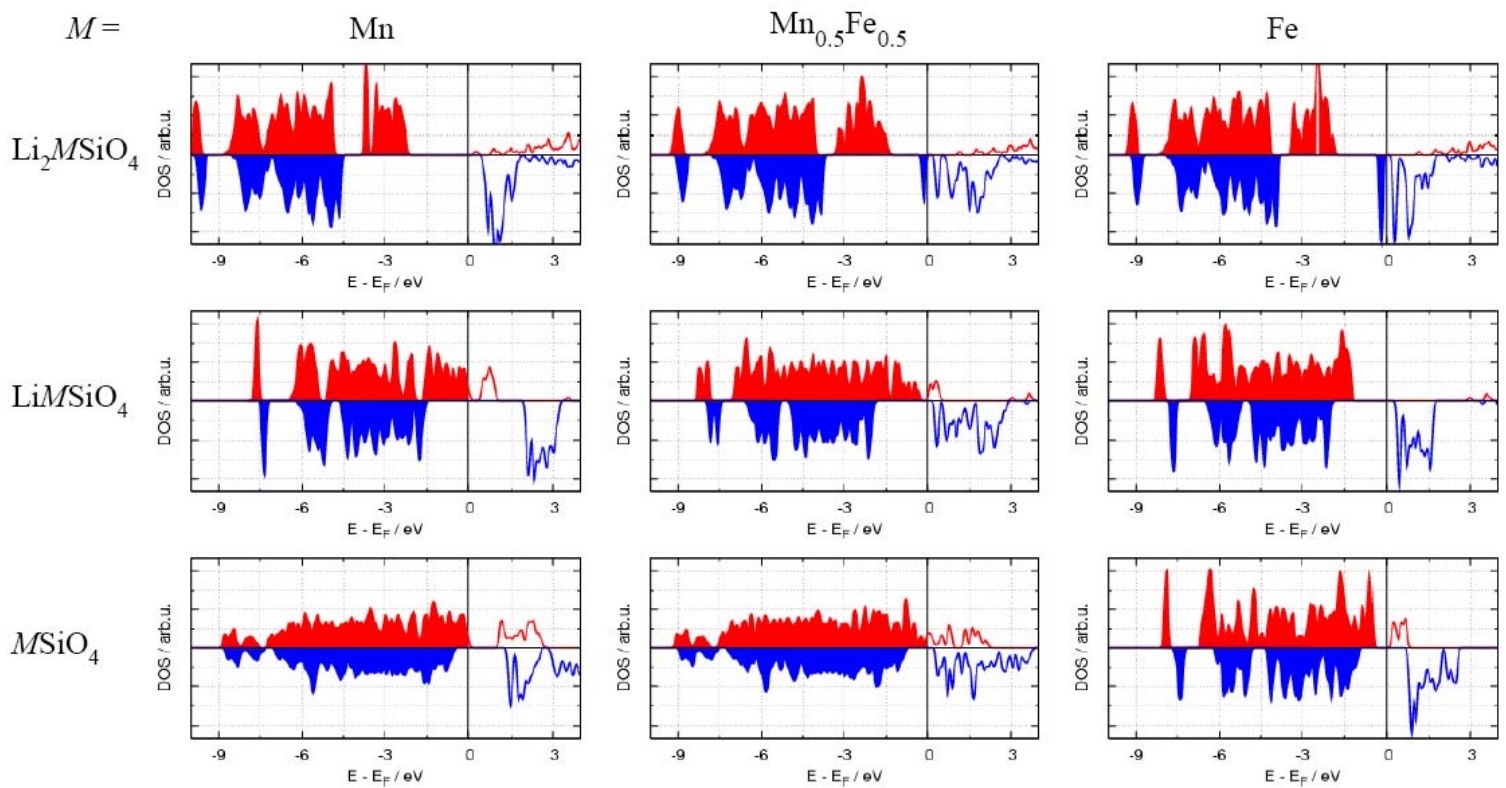

Figure S5. GGA calculated spin up and down density of states (DOS) for $\mathrm{Li}_{2 \mathrm{y}} M \mathrm{MSiO}_{4}, M=\mathrm{Mn}$ (left column), $\mathrm{Mn}_{0.5} \mathrm{Fe}_{0.5}$ (middle) and $\mathrm{Fe}$ (right), with various concetration of Lithium, $y=1,1 / 2$ and 0 . For fully delithiated $\mathrm{MnSiO}_{4}$ and $\mathrm{Mn}_{0.5} \mathrm{Fe}_{0.5} \mathrm{SiO}_{4}$ the DOS of the collapsed (see text) structures are shown. 
a)

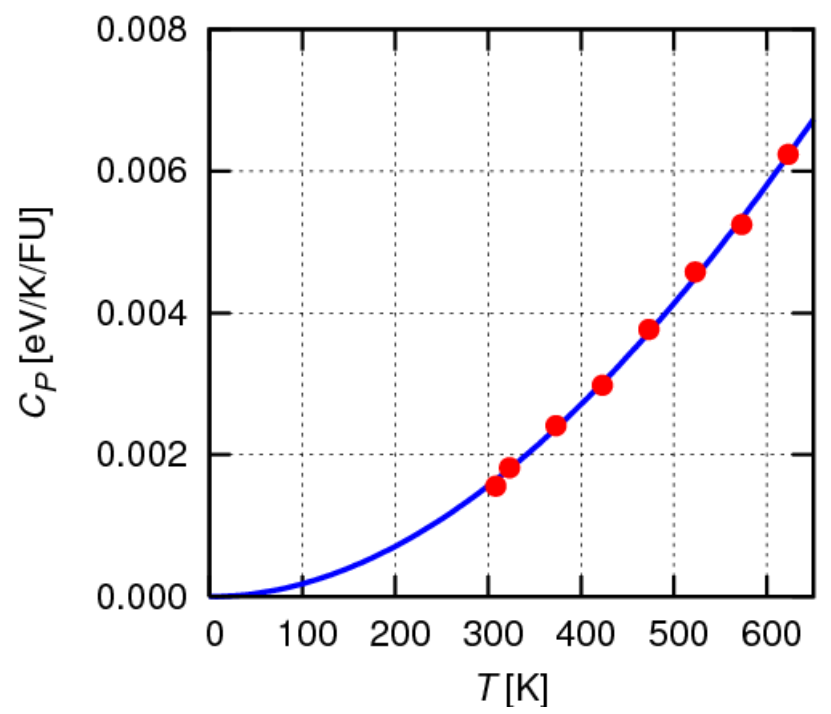

(b)

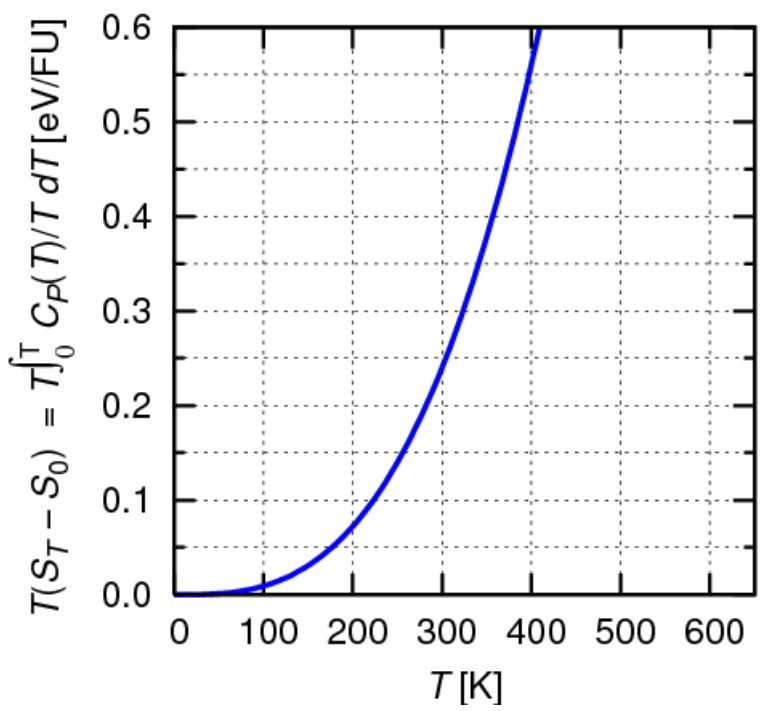

Figure S6. (a) Measured heat capacities (points) fitted with the polynomial, $C_{P}(T)=a T^{2}+b T^{3}$. (b) Resulting $T\left(S_{T}-S_{0}\right)=T \int_{0}^{T} C_{P}(T) / T d T$ as a function of $T$. 